INPLASY

PROTOCOL

To cite: Sacco et al. 18 years of medication related osteonecrosis of the jaw

(MRONJ) research: where are we now? - An umbrella review. Inplasy protocol 202160061. doi:

10.37766/inplasy2021.6.0061

Received: 18 June 2021

Published: 18 June 2021

Corresponding author:

Roberto Sacco

roberto.sacco@manchester.ac.uk

Author Affiliation:

The University of Manchester, Division of Dentistry, School of Medical Sciences, Oral Surgery Department, Manchester, UK.

Support: No Financial Support.

Review Stage at time of this submission: Data extraction.

Conflicts of interest:

None declared.

\section{8 years of medication related osteonecrosis of the jaw (MRONJ) research: where are we now? - An umbrella review}

Sacco, $\mathrm{R}^{1}$.

Review question / Objective: What is the current state of evidence related to MRONJ after 18 years of study? Population (P): any (no limits of age) patients with MRONJ Interventions (I): any types - Comparison (C): any types Outcome ( $(0)$ : state of knowledge based on the type of studies included in the reviews - Study (S): systematic review (SR) or meta-analysis (MA).

Condition being studied: Medication-related osteonecrosis of the jaw (MRONJ) is an irreversible adverse event related principally to antiresorptive medications (e.g. bisphosphonates and receptor activator of nuclear factor Kappa-B ligand inhibitors) and angiogenesis inhibitors. These type of drug therapies are used for the treatment of the skeletal manifestation of malignancies and/or bone metastases, and in the management of osteoporosis, Paget's disease or hypercalcaemia. The pathogenesis of MRONJ remains unclear, however a number of risk factors have been identified that are associated with an increased likelihood of MRONJ development. Many additional factors have been reported in the literature as being associated with accelerated development and/or increased severity of the condition, but for most of these it remains unclear whether or not they are causative factors.

INPLASY registration number: This protocol was registered with the International Platform of Registered Systematic Review and Meta-Analysis Protocols (INPLASY) on 18 June 2021 and was last updated on 18 June 2021 (registration number INPLASY202160061).

\section{INTRODUCTION}

Review question / Objective: What is the current state of evidence related to MRONJ after 18 years of study? - Population (P): any (no limits of age) patients with MRONJ - Interventions (I): any types - Comparison (C): any types - Outcome (O): state of knowledge based on the type of studies included in the reviews - Study (S): 
systematic review (SR) or meta-analysis (MA).

Condition being studied: Medicationrelated osteonecrosis of the jaw (MRONJ) is an irreversible adverse event related principally to antiresorptive medications (e.g. bisphosphonates and receptor activator of nuclear factor Kappa-B ligand inhibitors) and angiogenesis inhibitors. These type of drug therapies are used for the treatment of the skeletal manifestation of malignancies and/or bone metastases, and in the management of osteoporosis, Paget's disease or hypercalcaemia. The pathogenesis of MRONJ remains unclear, however a number of risk factors have been identified that are associated with an increased likelihood of MRONJ development. Many additional factors have been reported in the literature as being associated with accelerated development and/or increased severity of the condition, but for most of these it remains unclear whether or not they are causative factors.

\section{METHODS}

Search strategy: A search strategy for all databases was developed as follows: 1. Osteonecrosis [MeSH Terms] OR Avascular osteonecrosis of the jaw [MeSH Terms] OR Osteonecrosis of the jaw [MeSH Terms] OR MRONJ [MeSH Terms] OR ONJ [MeSH Terms] OR BONJ [MeSH Terms] OR ARONJ [MeSH Terms] OR BRONJ | 2. Patients [MeSH Terms] OR Any patients [MeSH Terms] OR Oncology [MeSH Terms] OR Osteoporosis [MeSH Terms] OR Nononcologic patients | 3. Systematic review [MeSH Terms] OR Review [MeSH Terms] OR Meta-analysis | 4. 1 and 2 and 3.

Participant or population: Any (oncology and non-oncology, no limits of age) patients with MRONJ.

Intervention: Any types.

Comparator: Any types.

Study designs to be included: Systematic review (SR) or meta-analysis (MA).
Eligibility criteria: The search strategy for this study will be focused on published systematic reviews and/or meta-analysis. No language restrictions will be imposed on the search. Narrative reviews, reviews not following PRISMA guidelines (after 2009), reviews without registration, animal reviews, and those reviews which included patients with a previous history of radiation therapy to the head and/or neck regions will be excluded.

Information sources: PubMed, MEDLINE, EMBASE and CINAHL.

Main outcome(s): Evaluate the current state of knowledge regarding the medication related osteonecrosis of the jaw as it relates with non-interventional type of studies and interventional type of studies, as well as the trends (number of SR and MA) per year.

Additional outcome(s): Evaluate factors such as: - Type of studies included in the reviews - Number of patients included in the review - Patients demographic - Type of patient groups (Oncology VS Nononcology).

Quality assessment / Risk of bias analysis: All selected papers will be carefully read by four independent review authors (RS, OA, JW and JY) and data will be extracted using a research report form. The number of included studies, design of the studies, number of patients, results, quality of the evidence and recommendation will be recorded. In case of missing information, authors will be contacted and given 6 weeks to respond.

Strategy of data synthesis: Following the screening of the eligible studies, all selected papers will be carefully read to identify study and to assess primary and secondary outcomes. Data will be extracted from each study and analysed. This will be done independently by selected authors. Where pooling of results is inappropriate, the results will be reported as narrative descriptions using a detailed commentary. 
Subgroup analysis: All participants will be included in the final analysis. If data permits, a subgroup analysis will be included in this review.

Sensitivity analysis: If sufficient data are extracted, a sensitivity analysis will be conducted to check the stability of the outcome results by excluding low methodological quality or high risk of bias studies.

Language: No language restrictions will be applied.

Country(ies) involved: United Kingdom.

Keywords: osteonecrosis; medication related osteonecrosis of the jaw; systematic review; umbrella review; evidence base medicine.

Contributions of each author:

Author 1 - Roberto Sacco. 